\title{
Long noncoding RNA DANCR, working as a competitive endogenous RNA, promotes ROCK1-mediated proliferation and metastasis via decoying of miR-335-5p and miR-1972 in osteosarcoma
}

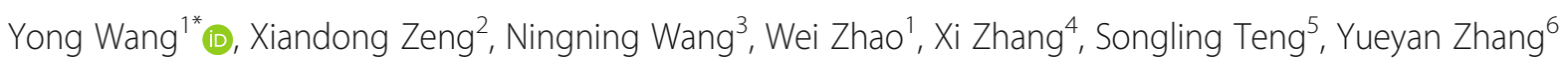
and Zhi Lu ${ }^{7}$

\begin{abstract}
Background: Accumulating evidences indicate that non-coding RNAs (ncRNAs) including long non-coding RNAs (IncRNAs) and microRNAs (miRNAs) acting as crucial regulators in osteosarcoma (OS). Previously, we reported that Rho associated coiled-coil containing protein kinase 1 (ROCK1), a metastatic-related gene was negatively regulated by microRNA-335-5p (miR-335-5p) and work as an oncogene in osteosarcoma. Whether any long non-coding RNAs participate in the upstream of miR-335-5p/ROCK1 axial remains unclear.

Methods: Expression of differentiation antagonizing non-protein coding RNA (DANCR) and miR-335-5p/miR-1972 in osteosarcoma tissues were determined by a qRT-PCR assay and an ISH assay. Osteosarcoma cells' proliferation and migration/invasion ability changes were measured by a CCK-8/EDU assay and a transwell assay respectively. ROCK1 expression changes were checked by a qRT-PCR assay and a western blot assay. Targeted binding effects between miR-335-5p/miR-1972 and ROCK1 or DANCR were verified by a dual luciferase reporter assay and a RIP assay. In vivo experiments including a nude formation assay as well as a CT scan were applied to detect tumor growth and metastasis changes in animal level.
\end{abstract}

Results: In the present study, an elevated DNACR was found in osteosarcoma tissue specimens and in osteosarcoma cell lines, and the elevated DNACR was closely correlated with poor prognosis in clinical patients. Functional experiments illustrated that a depression of DANCR suppressed ROCK1-mediated proliferation and metastasis in osteosarcoma cells. The results of western blot assays and QRT-PCR assays revealed that DANCR regulated ROCK1 via crosstalk with miR-335-5p and miR-1972. Further cellular behavioral experiments demonstrated that DNACR promoted ROCK1meidated proliferation and metastasis through decoying both miR-335-5p and miR-1972. Finally, the outcomes of in vivo animal models showed that DANCR promoted tumor growth and lung metastasis of osteosarcoma.

Conclusions: LnCRNA DANCR work as an oncogene and promoted ROCK1-mediated proliferation and metastasis through acting as a competing endogenous RNA (ceRNA) in osteosarcoma.

Keywords: LncRNA DANCR, miR-335-5p/miR-1972, Osteosarcoma, Proliferation/Metastasis, ceRNA

\footnotetext{
*Correspondence: wy_landy1116@163.com

Yong Wang and Xiandong Zeng are co-first authors.

${ }^{1} 4$ th Department of Orthopedic Surgery, Central Hospital affiliated to

Shenyang Medical College, No.5, South Seven West Road, Tiexi District,

Shenyang, Liaoning 110024, People's Republic of China

Full list of author information is available at the end of the article
}

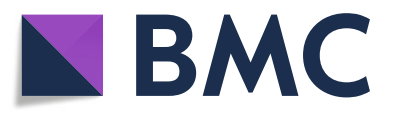

(c) The Author(s). 2018 Open Access This article is distributed under the terms of the Creative Commons Attribution 4.0 International License (http://creativecommons.org/licenses/by/4.0/), which permits unrestricted use, distribution, and reproduction in any medium, provided you give appropriate credit to the original author(s) and the source, provide a link to the Creative Commons license, and indicate if changes were made. The Creative Commons Public Domain Dedication waiver (http://creativecommons.org/publicdomain/zero/1.0/) applies to the data made available in this article, unless otherwise stated. 


\section{Background}

Osteosarcoma (OS) is a prevalent malignant bone tumor whose predictive sites are metaphysis of long bones among adolescents [1]. The character of fast growth and early stage of metastasis takes the main charge for the unfavorable prognosis of osteosarcoma [2]. As a result of its vicious biological behavior, being present of pulmonary metastasis is approximately $20 \%$ in the first visit of patients [3]. Consequently, seeking out a crucial metastatic-related molecular and identifying its underlying mechanism in osteosarcoma are compelling needed.

Long non-coding RNAs (lncRNAs), which are a group of transcribed RNA molecules and with length of more than 200 nucleotides, are involved in diverse biological processes in tumor initiation, growth and metastasis through epigenetic, transcriptional and post transcriptional mechanisms [4-6]. IncRNAs present multiple functions including posttranscriptional regulation, chromatin modification in various malignant tumors such as lung adenocarcinoma, esophageal squamous cell carcinoma, renal cell carcinoma, hepatocellular carcinoma and osteosarcoma [7-11]. Among the working mechanisms of lncRNAs, competing endogenous RNA (ceRNA) theory which was first proposed by Leonardo Salmena received high recognition in noncoding RNA area worldwide [12]. CeRNA theory hypothesizes that RNA transcriptions includes lncRNAs communication through a new "language" mediated by microRNA response elements (MREs).

Previous studies by our group indicated that miR-335 functioned as a tumor suppressor by way of directly targeting to its downstream gene - Rho associated coiled-coil containing protein kinase 1 (ROCK1) [13, 14]. Here, we wondered whether lncRNA could interact with miR-335 via the mechanism of ceRNA. Online prediction driving differentiation antagonizing non-protein coding RNA (DANCR) - a member of lncRNAs - to arouse our attention for its similar MREs which might be provided for miR-335-5p as ROCK1 did. Even further, we found that DANCR and ROCK1 shared two MREs for two miRNAs, miR-335-5p and miR-1972, respectively. In the present study, we focused on the ceRNA network which is comprising of DANCR, miR-335-5p/ miR-1972 and ROCK1, as well as explored the potential effect of DANCR to ROCK1 - mediated proliferation and migration/invasion in osteosarcoma.

\section{Methods}

\section{Patients and tissue samples}

Ninety-five cases of osteosarcoma tissues and matched para-tumor tissues used in this study were collected under the permissions of patients during tumorectomy in Liaoning Cancer Hospital \& Institute from February 2013 to September 2017. None of the patients had received chemotherapy before surgery and all the 95 cases were diagnosed based on a definite pathological diagnosis and the clinical stages of these patients were determined according to the tumor.node.metastasis (TNM) classification of the International Union Against Cancer (UICC). Written informed consent was obtained from all participants. The Institute Research Medical Ethics Committee of Central Hospital affiliated to Shenyang Medical College and Liaoning Cancer Hospital \& Institute granted approval for this study.

\section{Cell culture}

Human osteosarcoma cell lines MG-63, U2OS, MNNG/ HOS, 143B and human osteoblast cell line hFOB 1.19 were purchased from the Chinese Academy of Sciences Cell Bank (Shanghai, China) and maintained in DMEM/ F12 (Gibco, El Paso, TX, USA), DMEM (Gibco), DMEM, MEM, (Gibco) and DMEM, respectively. All mediums were supplemented with $10 \%(\mathrm{v} / \mathrm{v})$ fetal bovine serum (FBS, Sigma, St. Louis, MO, USA), $100 \mathrm{IU} / \mathrm{ml}$ penicillin (Baomanbio, China) and $100 \mathrm{mg} / \mathrm{ml}$ streptomycin (Baomanbio, China). All osteosarcoma cell lines were cultured at $37{ }^{\circ} \mathrm{C}$ in a humidified atmosphere containing $5 \% \quad \mathrm{CO}_{2}$ while human osteoblast cell line hFOB 1.19 was cultured at $34{ }^{\circ} \mathrm{C}$ with $5 \% \mathrm{CO}_{2}$.

\section{Plasmids and cell transfection}

MiR-335-5p mimics, miR-1972 mimics, NC mimic, miR335-5p inhibitor, miR-1972 inhibitor and NC inhibitor were purchased from Riobio (Ribobio, Guangzhou, China). DANCR overexpression plasmids pcDNA-DANCR, wtDNACR-335, wt-DANCR-1972, mut-DANCR-335 and mut-DANCR-1972 as well as DANCR and ROCK1 knockdown plasmids DANCR shRNA and ROCK1 shRNA with a corresponding negative control shRNA (NC shRNA) were chemically synthesized by GenePharma (GenePharma, Shanghai, China). The sequence of DANCR shRNA and ROCK1 shRNA were listed in Additional file 1: Table S1. Quantitative real-time PCR analysis was applied to detect DANCR and ROCK1 expression level to value the silenced efficiencies. The results were shown in Additional file 2: Figure S2A, DANCR shRNA - 01 was selected as the optimum shRNA in the subsequent experiments due to the highest suppressive effect. ROCK1 shRNA-01 was selected by the same means as presented in Additional file 2: Figure S2B and was employed in following ROCK1 RNAi experiment. The aforementioned plasmids were transfected into MNNG/HOS and 143B cells by Lipofectamine 2000 (Invitrogen, Carlsbad, CA, USA) according to the manufacturer's protocol. For a stable cell transfection, cells were selected by the culture medium containing $0.4 \mathrm{mg} / \mathrm{ml}$ Geneticin (G418; Invitrogen). After 6 weeks, G418-resistant cell clones were established. 
Reverse transcription and quantitative real-time PCR

The procedure was carried out as previously described [15]. Total RNAs were extracted using Trizol reagent (Invitrogen, Carlsbad, CA, USA). cDNAs were synthesis by PrimeScriptTM RT reagent kit (Takara, Dalian, China). qPCR was performed with the SYBR Premix Ex Taq II kit (TaKaRa) and the Applied Biosystems 7500 Fluorescent Quantitative PCR system (Applied Biosystems Life Technologies, USA). GAPDH and U6 were used to normalize the expression levels of the DANCR/ ROCK1 and miR-335-5p/miR-1972, respectively. Primers sequences were listed in Additional file 1: Table S1.

\section{Western blot analysis}

Total proteins were isolated using radio immunoprecipitation assay (RIPA) lysis buffer (Sigma, St. Louis, MO, USA) and qualified by BCA detecting kit (Keygen, Nanjing, China) flollowing to the manufacture's protocol. Proteins samples were subjected to $10 \%$ SDS-PAGE and transferred onto a PVDF membrane, then incubated with ROCK1 (Abcam, Cambridge, MA, UK; dilution rates of 1:2000) and GAPDH antibodies (Abcam, dilution rates of $1: 500)$ at $4{ }^{\circ} \mathrm{C}$ overnight, respectively. The next day, the membranes were incubated with secondary antibodies (Abcam, dilution rates of 1:2000) at room temperature for $1 \mathrm{~h}$. Protein bands were detected on X-ray film via enhanced chemiluminescence detection system.

\section{Cell proliferation assays}

Osteosarcoma cells' changes of proliferative capacity were determined by 5-Ethynyl-20-Deoxyuridine (EDU) incorporation assay and cell counting Kit-8 (CCK-8) assay. For EDU incorporation assay, the procedure was carried out according to the manufacturer's instructions with EDU detection kits (Keygen, Nanjing, China). CCK8 assay was executed as previously reported [16]. In brief, osteosarcoma cells were seeded in 96-well plates $\left(2 \times 10^{3}\right)$ supplemented with complete growth medium and followed by different transfection $24 \mathrm{~h}$ later. At days 1, 2, 3, 4 and 5 after transfection, $10 \mu \mathrm{l} \mathrm{CCK-8} \mathrm{solution}$ was added into each well and incubated for $2 \mathrm{~h}$. The absorbance was measured at an optical density of $450 \mathrm{~nm}$ using Microplate reader (Bio-Rad, CA, USA). Experiments were repeated in triplicate.

\section{Transwell assays}

The procedure was carried out as previously described [5]. In short, MNNG/HOS and 143B cells were seeded on uncoated (for migration assays) and Matrigel-coated (for invasion assays) upper chambers (BD Bioscience, New Jersey, USA) respectively. Culture medium noncontaining and containing 10\% FBS were supplemented into the upper and lower wells respectively and incubated for further $24 \mathrm{~h}$. Followed by wiping of non- migrated or non-invaded cells. Then the filters were fixed in $90 \%$ ethanol and followed by crystal violet staining. Five random fields were counted per chamber by using an inverted microscope (Olympus, Tokyo, Japan).

\section{In situ hybridization assay and immunohistochemistry analysis}

In situ hybridization assay was performed on fresh osteosarcoma tissue slices. In brief, slices were washed with $1 \times$ phosphate buffered saline (PBS) containing $0.5 \%$ Triton X-100, afterwards be incubated with appropriate amount of anti-DANCR, anti-miR-335-5p and anti-miR-1972 oligodeoxy-nucleotide probes (RiboBio, Guangzhou, China) with hybridization solution containing $1 \%$ blocking solution in humid chamber at $37{ }^{\circ} \mathrm{C}$ overnight. The next day, slices were then washed three times for 5 min each at $42{ }^{\circ} \mathrm{C}$ with $0.1 \%$ Tween- 20 in $4 \times$ sodium citrate buffer (SSC), once for $5 \mathrm{~min}$ in $2 \times$ SSC and once for $5 \mathrm{~min}$ in $1 \times$ SSC in dark. Following, after be rinsed with $1 \times$ PBS for 5 min triply at room temperature and be re-stained by DAPI (Cell Signaling Technologies, Danvers, USA), fluorescent microscope (Leica, Wetzlar, Germany) was applied to observe and photograph the slices. Images were analyzed using Image - Pro Plus 6.0 software (Media Cybernetics, Rockville, USA).

The procedure of immunohistochemistry was performed as previously described [16].

\section{Dual luciferase reporter assay}

The procedure was carried out as previously described [10]. Wild and mutant reporter plasmids of DANCR (wtDANCR-335-luc, mut-DANCR-335-luc, wt-DANCR-1972luc and mut-DANCR-1972-luc) and ROCK1 (wt-ROCK1335-luc, mut-ROCK1-335-luc, wt-ROCK1-1972-luc and mut-ROCK1-1972-luc) which containing a wild or mutant miR-335-5p or miR-1972 binding sites were individually synthesized by GenePharma (GenePharma, Shanghai, China). When HEK293 cells achieved to 70\% confluence, the synthesized reporter plasmids were co-transfected with miR-335-5p mimics/miR-1972 mimics or mimic control respectively by Lipofectamine 2000 (Invitrogen). Fortyeight hours later, the changes of the fluorescence in each group were detected using Dual-Luciferase Reporter Assay System (Promega, Madison, WI, USA) according to the manufacturer's protocol.

\section{RNA immunoprecipitation (RIP) assay}

The procedure was carried out as previously described using Magna RNA-binding protein immunoprecipitation kit (Millipore, Billerica, MA, USA) [17]. In brief, whole-cell lysate was incubated with RIP buffer containing magnetic beads conjugated with human anti-Ago2 antibody, or normal mouse IgG as negative control. Samples were incubated with proteinase $\mathrm{K}$ and then immunoprecipitated 
RNA was isolated. The RNA concentration was measured by spectrophotometer (Thermo Scientific, Waltham, MA, USA) and the RNA quality was assessed with bio-analyzer (Agilent, Santa Clara, CA, USA). Furthermore, purified RNAs were extracted and analyzed by quantitative realtime PCR to demonstrate the presence of binding targets.

\section{In vivo nude mouse models}

Female nude mice (4-5 weeks old) were purchased from Animal Care and Use Committee of Dalian Medical University Ltd., and kept under sterile specific-pathogenfree (SPF) conditions. $1 \times 10^{6} 143 \mathrm{~B}$ cells (mixed with Matrigel, BD Bioscience, 1:1) with stable overexpression of DANCR (DANCR puro) and with corresponding blank vector (pMSCV puro) were injected subcutaneously or intravenously for the determination of tumor growth and metastasis. Tumor formation and metastasis were monitored by Fluorescent Imager (IVIS Spectrum, Caliper Life Sciences, Hopkinton, MA, once per week for 4 weeks) and Computed Tomography (CT, Siemens, München, Germany, at week four) scan, respectively. The subcutaneous formatted tumor nodes and the lungs from metastatic group were harvested for further detection. This study was carried out in accordance with the Guide for the Care and Use of Laboratory Animals of the National Institutes of Health and was granted by the Institute Research Medical Ethics Committee of Central Hospital affiliated to Shenyang Medical College.

\section{Statistical analysis}

The significance of differences between groups was assessed by Student's t-test, one-way ANOVAs or $\chi^{2}$ test, as appropriate. Survival curves were estimated by the Kaplane-Meier method. The log-rank test was used to determine the statistical differences between survival curves. All statistical analyses were performed using GraphPad Prism V5.0 (GraphPad Software, Inc., La Jolla, CA, USA) and SPSS 19.0 (IBM, SPSS, Chicago, IL, USA). A two-tailed $P<0.05$ was considered as statistically significant while $P<0.01$ was very significant.

\section{Results}

DANCR was elevated in osteosarcoma tissues and cell lines and was correlated with poor prognosis in osteosarcoma patients

The expression of DANCR in collected 95 cases of osteosarcoma tissues and matched para-tumor tissues were determined by applying of quantitative real-time PCR (qRT-PCR) as normalizing to GAPDH. As the results presented in Fig. 1a, DANCR was elevated in most osteosarcoma tissues $(72 / 95,75.79 \%)$ when compared with para-tumor tissues. Additionally, the potential correlation between expression level of DANCR and clinicopathological features were uncovered. As the outcomes displayed in
Fig. 1b-d, the expression of DNACR was notably higher in patients with advanced stage, lymph node metastasis and distant metastasis. Meanwhile, further analysis by Pearson Chi-Square test or Fisher's Exact Test indicated higher expression of DANCR was correlated with bigger tumor size $(\geq 5 \mathrm{~cm})$, advanced staging (IIB/III) and distant metastasis (Table 1). Moreover, In situ hybridization (ISH) analysis was applied to evaluate the expression of DANCR in tissue level. As shown in Fig. 1e, DANCR was gradually strongly stained with staging in osteosarcoma tissues than that in para-tumor tissues. Also, DANCR was intensively stained in osteosarcoma cells' cytoplasm. Even further, the expression level of DANCR in 4 osteosarcoma cell lines MG-63, U2OS, MNNG/HOS, 143B and a normal human osteoblastic cell line hFOB 1.19 were also measured by qRT-PCR. As was illustrated in Fig. 1f, the expression of DANCR was significantly elevated in osteosarcoma cell lines MG-63, U2OS, MNNG/HOS, 143B compared to that in hFOB 1.19. Finally, Kaplan-Meier analysis and logrank test were used to evaluate the correlation between the elevated DANCR and the final survival time of osteosarcoma patients. The results in Fig. 1g suggested that high DANCR expression was inversely correlated with osteosarcoma patients' overall survival.

\section{DANCR promoted proliferation and migration/invasion in MNNG/HOS and 143B cells}

Since DANCR was highly expressed in osteosarcoma and was correlated with tumor size and distant metastasis in clinical cases, we wondered about the function DANCR might act on osteosarcoma cells' proliferation and metastasis. Up-regulation and down-regulation of DANCR by transfection of DANCR-shRNA and pcDNA-DANCR respectively were confirmed by qRT-PCR (Fig. 2a-b). Then, 5-ethynyl-2'-deoxyuridine (EdU) incorporation assays and CCK-8 assays were applied to evaluate the effects of DANCR on cell proliferation in osteosarcoma cells MNNG/HOS and 143B. As was presented in Fig. 2c-d, DANCR confidently enhanced the proliferative capacity in MNNG/HOS and 143B cells, and vice versa. In addition, the migration/invasion ability changes in MNNG/HOS and 143B cells were determined by transwell assay. As was shown in Fig. 2e-f, depression and elevation of DANCR also positively regulated osteosarcoma cells' migration/invasion.

\section{DANCR promoted proliferation and migration/invasion} via up-regulation of ROCK1 in MNNG/HOS and 143B cells Increasing evidences demonstrated that lncRNAs regulated various downstream genes to exert multiple functions. According to our and other previous researches, ROCK1 was closely related to osteosarcoma cells' proliferation and migration/invasion [5, 18]. Here, we attempted to figure out whether the regulation effect of DANCR on osteosarcoma cells' proliferation and migration/invasion was 


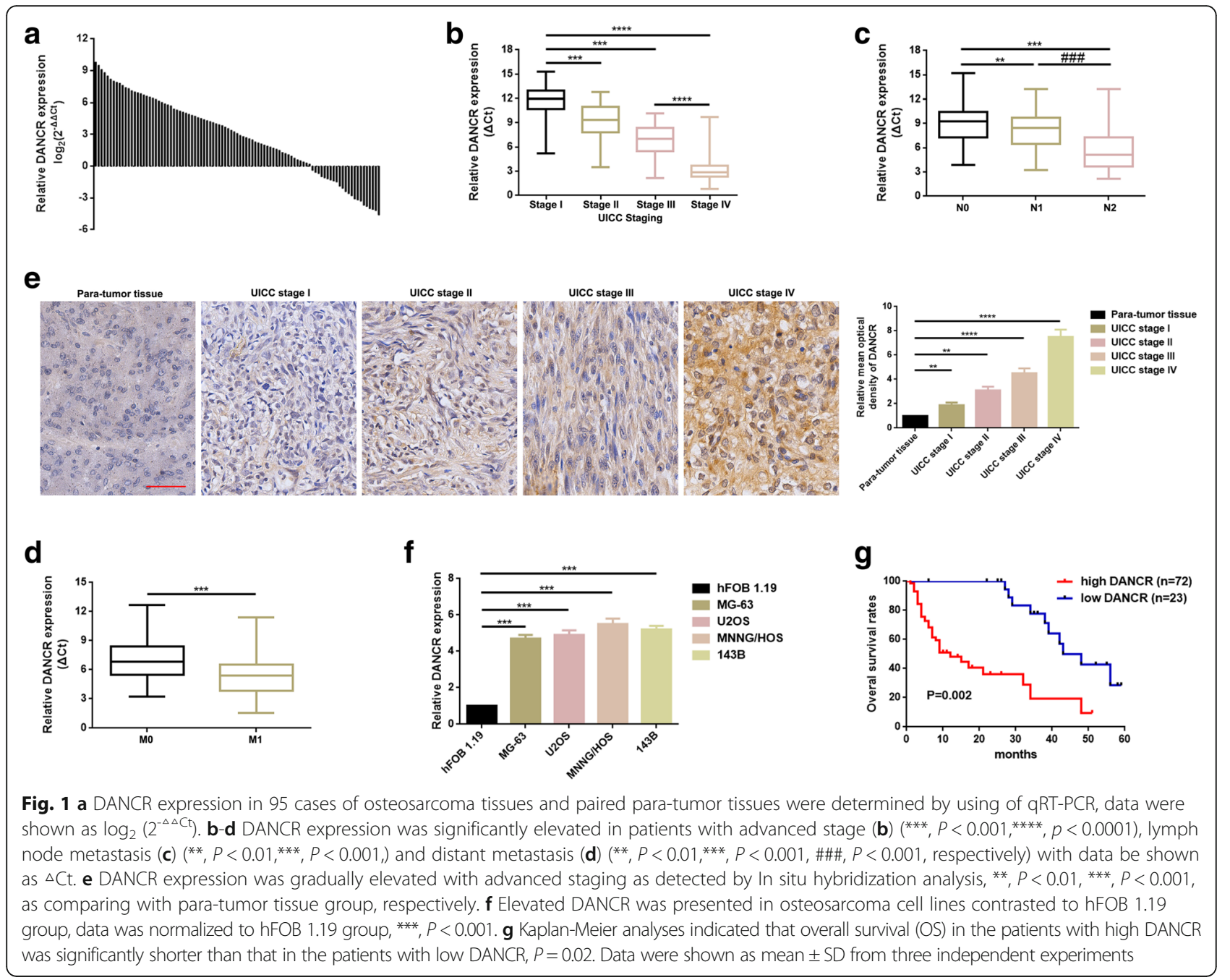

achieved through ROCK1. We firstly demonstrated that ROCK1 was elevated in osteosarcoma tissue specimens and in osteosarcoma cell lines (Fig. 3a-b). Also, we displayed the positive correlation between DANCR and ROCK1 (Fig. 3c). We then confirmed that up- and down-regulation of DANCR could positively affected ROCK1 expression both in mRNA and in protein level (Fig. 3d-e). Furthermore, we determined the role which ROCK1 might play in DANCRrelated proliferation and migration/invasion. As the results presented in Fig. 3f-g, knockdown of ROCK1 (co-transfection of pcDNA-DANCR and ROCK1 shRNA) attenuated the proliferating-assisted effect DANCR in MNNG/HOS and 143B cells. Meanwhile, transwell assay elucidate a similar tendency of ROCK1 performing on migration/invasion as it did on proliferation (Fig. 3h-i).

\section{DANCR up-regulated ROCK1 via crosstalk with miR-335-5p and $\mathrm{miR}-1972$}

Lately, researches on the competitive endogenous RNA (ceRNA) theory between IncRNAs and miRNAs are the hotspots in the field of noncoding RNAs. Therefore, we specuated whether DANCR might crosstalk with any potential miRNAs in dependence of the same mechanism in osteosarcoma. Firstly, an online lncRNA prediction software (DIANA-LncBase) was applied to predict the potential miRNAs that might interact with DANCR. Among the top 18 miRNAs of which the predictive binding scores were higher than 0.9 (Additional file 3: Figure S1), miR-335-5p and miR-1972 stroke our sight because DANCR and ROCK1 shared the similar miRNA response elements (MREs) for miR-335-5p and miR1972 (Fig. 4a, predicted by DIANA-LncBase and TargetScan). Secondly, the expression of miR-335-5p and miR-1972 in osteosarcoma tissues and cell lines were determined by ISH and qRT-PCR. As was shown in Fig. 4b-c, miR-335-5p and miR-1972 were lower expressed in osteosarcoma tissue and cell lines, individually. And this lower expression level of miR-335-5p and miR1972 indirectly provided the possibility that DANCR might co-work with them in the progression of osteosarcoma. 
Table 1 Association of DANCR expression with clinicopathological features of osteosarcoma

\begin{tabular}{|c|c|c|c|c|}
\hline \multirow[t]{2}{*}{ Features } & \multirow{2}{*}{$\begin{array}{l}\text { No. } \\
\text { of } \\
\text { cases }\end{array}$} & \multicolumn{2}{|c|}{ DANCR } & \multirow[t]{2}{*}{$p$ valuet } \\
\hline & & High & Low & \\
\hline Age at diagnosis & & & & 0.481 \\
\hline$<18$ & 52 & 40 & 12 & \\
\hline$\geq 18$ & 43 & 32 & 11 & \\
\hline Gender & & & & 0.472 \\
\hline Female & 51 & 38 & 13 & \\
\hline Male & 44 & 34 & 10 & \\
\hline Histological subtype & & & & 0.674 \\
\hline Osteoblastic & 9 & 6 & 3 & \\
\hline Chondroblastic & 18 & 12 & 6 & \\
\hline Fibroblastic & 38 & 30 & 8 & \\
\hline Mixed & 30 & 24 & 6 & \\
\hline Clinical stage & & & & 0.005 \\
\hline$I+\| A$ & 42 & 26 & 16 & \\
\hline$\|\mathrm{B} /\|$ & 53 & 46 & 7 & \\
\hline Distant metastasis & & & & 0.002 \\
\hline Absent & 37 & 22 & 15 & \\
\hline Present & 58 & 50 & 8 & \\
\hline Tumor size $(\mathrm{cm})$ & & & & 0.014 \\
\hline$<5$ & 39 & 23 & 16 & \\
\hline$\geq 5$ & 56 & 49 & 7 & \\
\hline Anatomic location & & & & 0.431 \\
\hline Tibia/femur & 49 & 38 & 11 & \\
\hline Elsewhere & 46 & 34 & 12 & \\
\hline
\end{tabular}

$+P$-value obtained from Pearson Chi-Square test or Fisher's Exact Test

Thirdly, inverse correlations between DANCR and miR335-5p as well as DANCR and miR-1972 were presented by Pearson correlation analysis in the collected 95 cases of osteosarcoma tissues (Fig. 4d-e). We then verified that increase or decrease DANCR negatively regulated miR-3355p and miR-1972 (Fig. 4f-g). Conversely, up-regulated and down-regulated miR-335-5p and miR-1972 also inversely affected in DANCR expression (Fig. 4h-i). These outcomes indicated that DNACR can "crosstalk" with miR-335-5p and miR-1972 in a reciprocal suppression manner. MiRNAs are well known to regulate targeted downstream genes by directly binding to the 3 ' untranslated regions (3'UTRs) [19-21]. So, we further proved that miR-335-5p and miR1972 could negatively regulate ROCK1 mRNA expression and that ROCK1 was a direct target of miR-335-5p and miR-1972 (Fig. 4j-m). Finally, miR-335-5p and miR-1972 mimics were used to detect the effect they might execute on DANCR-induced ROCK1 elevation. As we expected, opposite to NC mimic, miR-335-5p mimics and miR-1972 mimics attenuated DANCR induced ROCK1 elevation (Fig. 4n-o). Together, the evidence in this section demonstrated that DANCR promoted ROCK1 expression via "crosstalk" with miR-335-5p and miR-1972.

\section{DANCR decoyed miR-335-5p and miR-1972 to facilitate ROCK1-mediated proliferation and migration/invasion} In the above sections, we verified that DANCR could "crosstalk" with miR-335-5p and miR-1972 and promoted ROCK1-mediated proliferation and migration/invasion. In this section, we tried to elucidate whether the ceRNA mechanism do exist among DANCR, miR-3355p/miR-1972 and ROCK1. We had proved that DANCR affected miR-335-5p and miR-1972 with a reciprocal suppression manner in the previous section, we here applied a luciferase assay to detect the specific binding sites between DNACR and miR-335-5p/miR-1972. As the results showed in Fig. 5b-c, compare to NC mimic, cotransfection of miR-335-5p or miR-1972 mimics and wt-DANCR led to a prominent decrease of fluorescence. When the theoretical binding sites DANCR might provide for miR-335-5p or miR-1972 were mutated (cotranfection of miR-335-5p mimics and wt-DANCR-335 or cotransfection of miR-1972 mimics and wt-DANCR1972), the fluorescence was restrengthened. Further, as the outcomes of a RIP binding assay presented, the level of DANCR and miR-335-5p/miR-1972 was higher in anti-Ago2 group than that in anti-normal IgG group which indicating that DANCR and miR-335-5p/miR1972 were in the same RNA induced silencing complex (RISC) (Fig. 5d-e). Combined with the findings above, we believed that DANCR decoyed miR-335-5p and miR1972 to promote their common-target gene - ROCK1. Furthermore, an antisense experiment was executed to final support the hypothesis that DANCR improved ROCK1-mediated proliferation and migration/invasion via directly decoying of miR-335-5p and miR-1972 in osteosarcoma cells.

\section{Elevation of DANCR promoted tumorigenesis and lung metastasis of osteosarcoma in vivo}

In this section, orthotopic xenograft mouse models were introduced to finally confirm the facilitative effect DANCR conducts on tumor growth and lung metastasis of osteosarcoma. As was shown in Fig. 6a-b, overexpression of DNACR (DANCR puro) significantly promoted tumor growth of ostesoacoma as comparing with blank vector (pMSCV puro) group. Meanwhile, consequence that elevated DANCR but depressed miR-335-5p and miR-1972 in DANCR puro group was noticed by a qRTPCR detection (Fig. 6e-g). Also, ROCK1 expression in the formatted subcutaneous nudes were determined by IHC and western blot. Data presented in Fig. 6c implied elevation of DNACR could promote ROCK1 expression. Further, at the fourth week after caudal vein injection, the metastatic nudes in lungs were determined by $\mathrm{CT}$ 


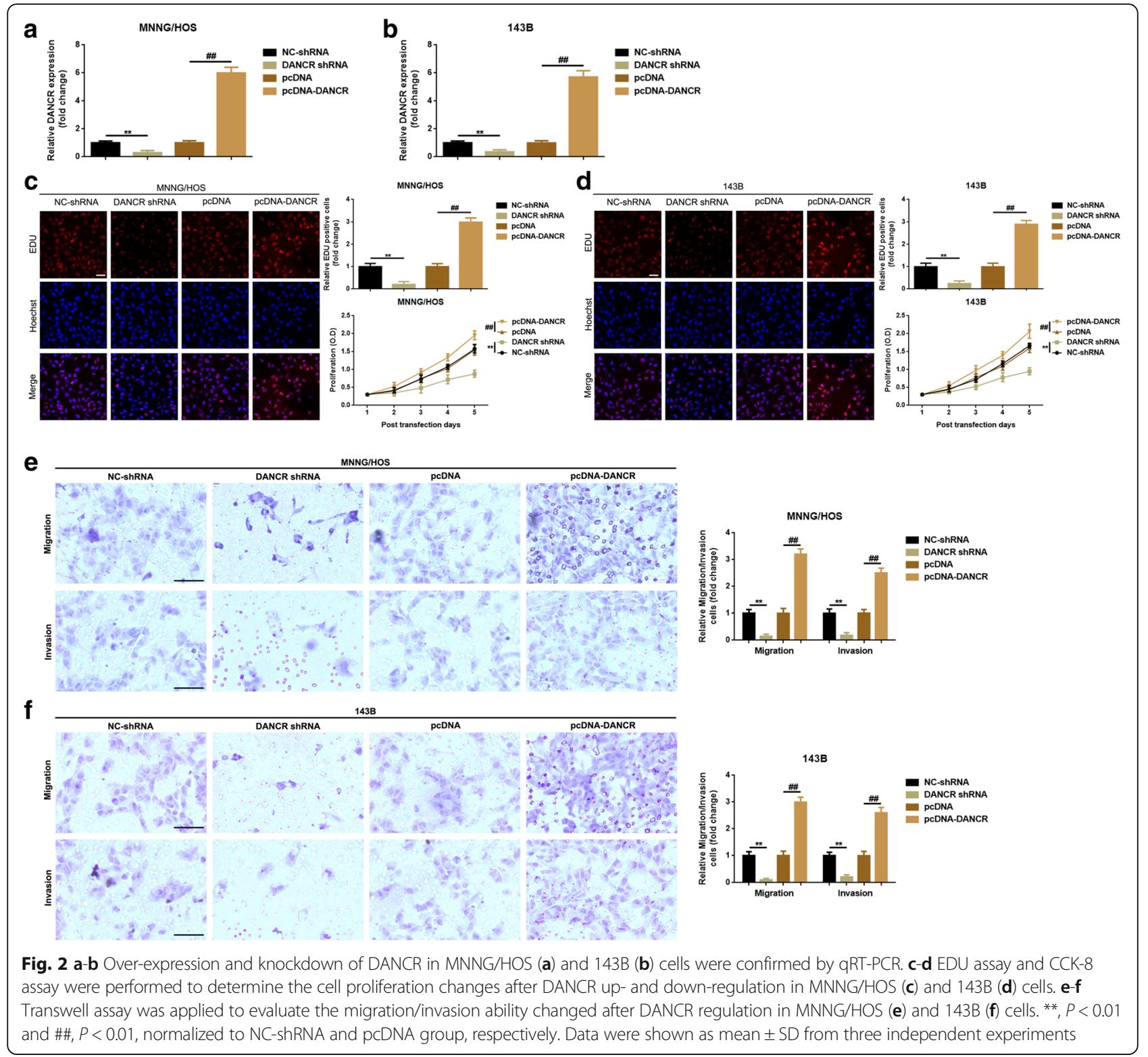

scan. As the representative images displayed in Fig. 6h, up-regulating DANCR also contributed to lung metastasis of osteosarcoma in mice. Finally, as the detective results of metastatic nodes in lung demonstrated in Fig. 6i-n, increasing DNACR boosted ROCK1 expression but inhibited miR-335-5p and miR-1972 expression. Together, the indication of this part confirmed that elevation of DANCR promoted tumorigenesis and lung metastasis of osteosarcoma in vivo.

\section{Discussion}

Recently, aberrant expression of some lncRNAs has been elucidated as functioning multiple effects in tumorgenesis of osteosarcoma [22-25]. DANCR is located in human chromosome $4 \mathrm{q} 12$, and is reported as an oncogene in diverse carcinomas. Yuan SX suggested that DANCR increased stemness features of hepatocellular carcinoma by derepression of catenin beta 1 (CTNNB1) [26]. Jia J persisted that DANCR promoted the invasion ability of C4-2B and CW22RV1 cells through epigenetically silencing TIMP2/3 expression in a research of prostate cancer [27]. To date, related researches on DNACR and osteosarcoma were rare. Jiang $\mathrm{N}$ revealed that DANCR could enhance tumor progression and cancer stemness features in osteosarcoma by upregulating AXL via miR33a-5p inhibition [28]. In the present study, we took DANCR as a study target based on the previous research foundation. We found that DANCR was raised in osteosarcoma cell lines and in osteosarcoma tissue specimens especially in specimens with late staging, lymph node 


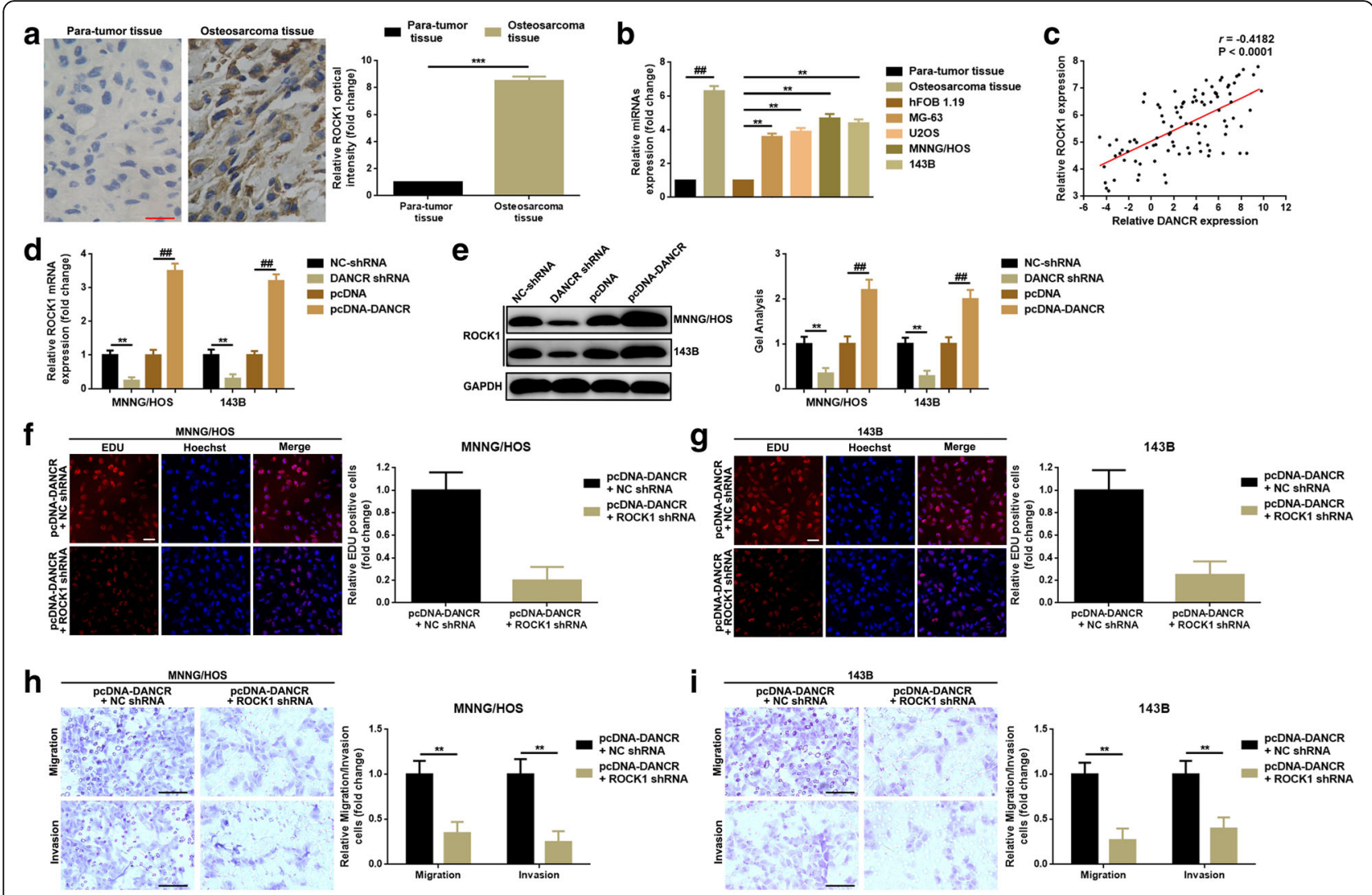

Fig. 3 a The elevated expression of ROCK1 in tissue level were displayed by an $I H C$ detection, ${ }^{* *}, P<0.001$, normalized to para-tumor tissue group. $\mathbf{b}$ ROCK1 was up-regulated in osteosarcoma tissue and cell lines as determined by a GRT-PCR, ${ }^{* *}, P<0.01$ and \#\#, $P<0.01$, normalized to para-tumor tissue group and hFOB 1.19 group, respectively. c A positive correlations between DNACR and ROCK1 was confirmed by a Pearson correlation analysis, $P<0.0001$. $\mathbf{d}$-e The expression of ROCK1 after DANCR regulation were measured by using of qRT-PCR (d) and western blot (e) in MNNG/HOS and 143B cells, separately. $\mathbf{f - g}$ RNAi technique was used to define the function ROCK1 might work on DANCR induced proliferation. Silencing ROCK1 by transfection of ROCK1 shRNA attenuated DANCR-induced facilitation of proliferation in MNNG/HOS (f) and 143B (g) cells, as determined by an EDU assay. $\mathbf{h}$-i A transwell assay was executed to verify the role of ROCK1 in DANCR-induced metastasis in MNNG/HOS (h) and 143B (i) cells. **, $P<0.01$, normalized to pcDNA-DANCR + NC shRNA group. Data were shown as mean \pm SD from three independent experiments

metastasis and distant metastasis. Also, we validated that elevated DANCR was closely correlated with a short survival time in patients. These data indicated that DANCR works as an oncogene in the progression of osteosarcoma. In the part of clinical detection, a total of 95 patients were involved in our research. The sample size is big enough to illustrate the role of DNACR playing in osteosarcoma. According to the outcomes of clinical detection, the further cellular level of EDU and transwell assays primarily reinforced that DANCR promoted osteosarcoma cells' proliferation and metastatic abilities. Therefore, exploring out the detailed mechanism of how DANCR working is meaningful.

LncRNAs are reported to regulate gene expression and chromatin structure via following ways: decoy effect, scaffold effect and post-transcriptional effect [29]. In 2011, the ceRNA theory was firstly proposed by Leonardo Salmena and was extensively accepted in noncoding RNA area. Numerous researches on ceRNA and cancers were explored [30-36]. In our previous studies, we found that miR-335-5p suppressed osteosarcoma cells' metastatic ability via targeting its downstream gene ROCK1 [14, 37]. ROCK1 was also broadly reported as a proliferation- and metastasis-related gene in various cancers [38-41]. Hence, we tried to elucidate whether DNACR could affect ROCK1 and its mediated proliferation and metastasis via a same mechanism as ceRNA. As the first encouraging result, we verified that DANCR did promote osteosarcoma cells' proliferation and metastasis via up-regulation of ROCK1. According to the previous researches, ROCK1 could be targeted by many miRNAs [41-44]. Also, it is well known that lncRNA can regulate numbers of miRNAs at the same time, and one miRNA can regulate an army of downstream target genes. We therefore wondered whether DNACR could co-work with one or more miRNAs to regulate ROCK1-mediated proliferation and metastasis. As a bright result, we found that DANCR and ROCK1 not only shared a similar 


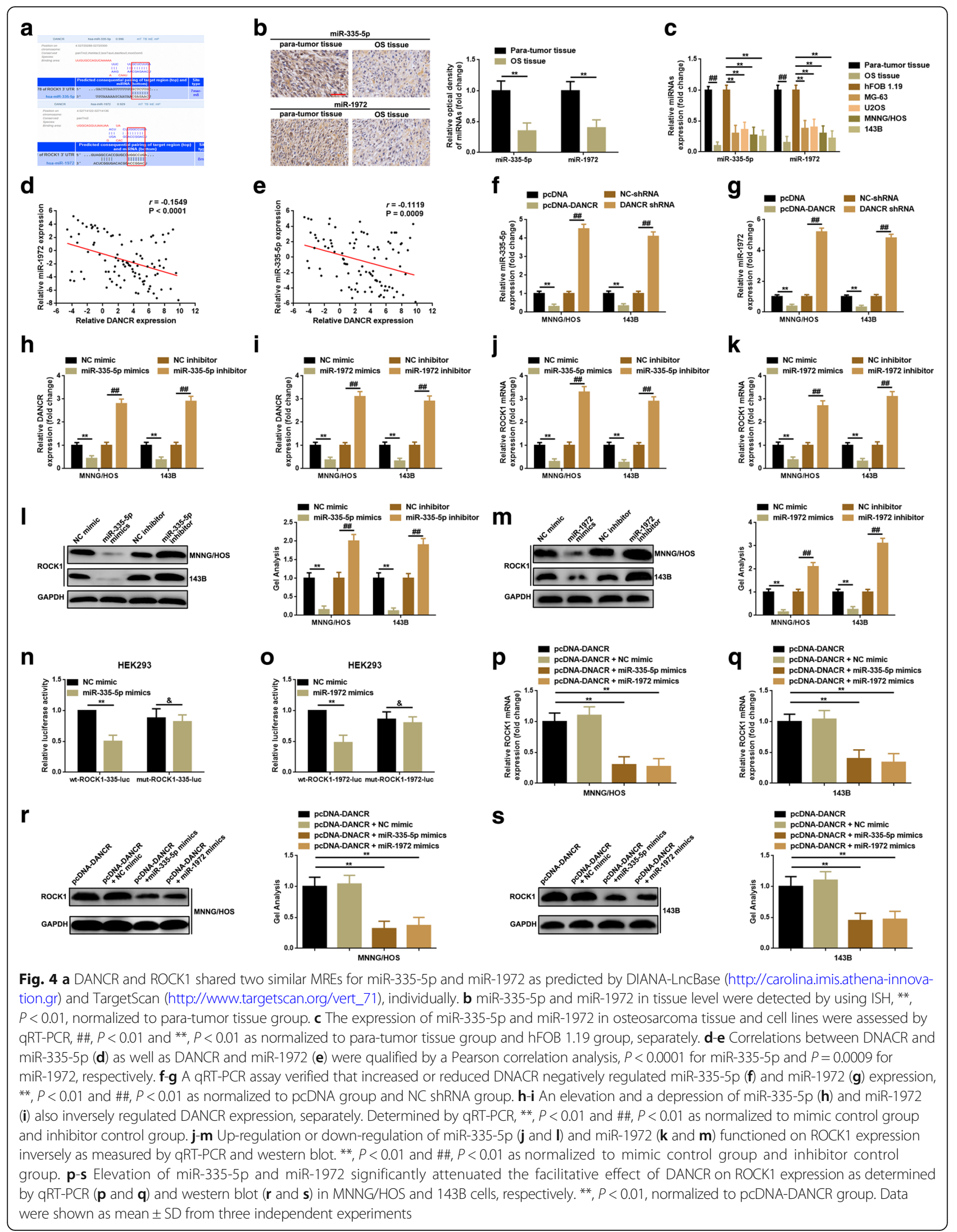




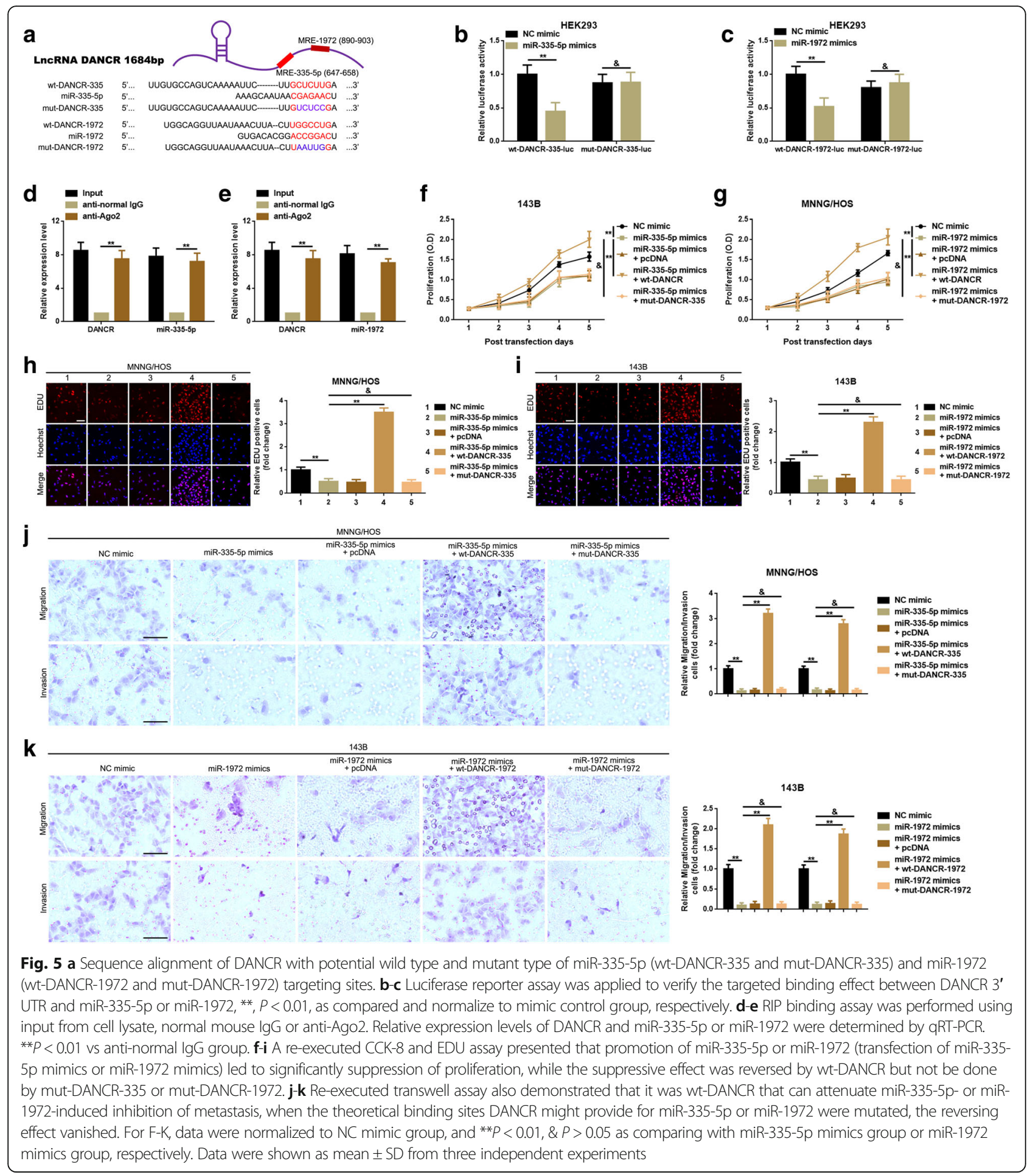

MREs for miR-335-5p but also shared another MREs for miR-1972. Meanwhile, we revealed the negative correlation between DNACR and miR-335-5p and miR-1972 by a Pearson correlation analysis, respectively. Further, a series of up and down functional experiments clear demonstrated the reciprocal suppressive effect between
DNACR and miR-335-5p and miR-1972 respectively which indicating that DANCR could crosstalk with these two miRNAs. It is widely accepted that miRNAs regulated their target genes by directly binding. We here designed a luciferase assay and a RIP binding assay to confirm the targeted binding effect between DANCR/ 


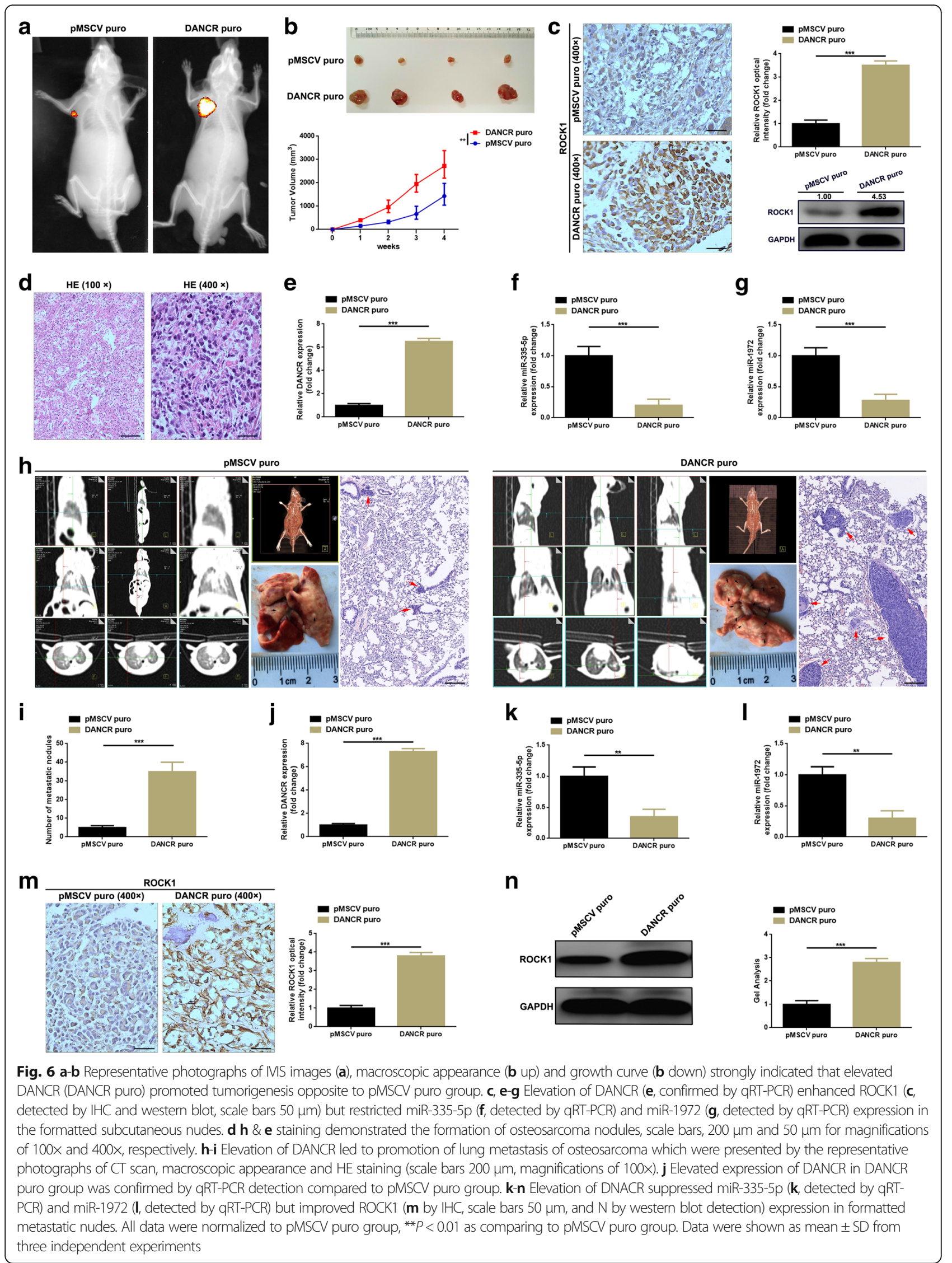




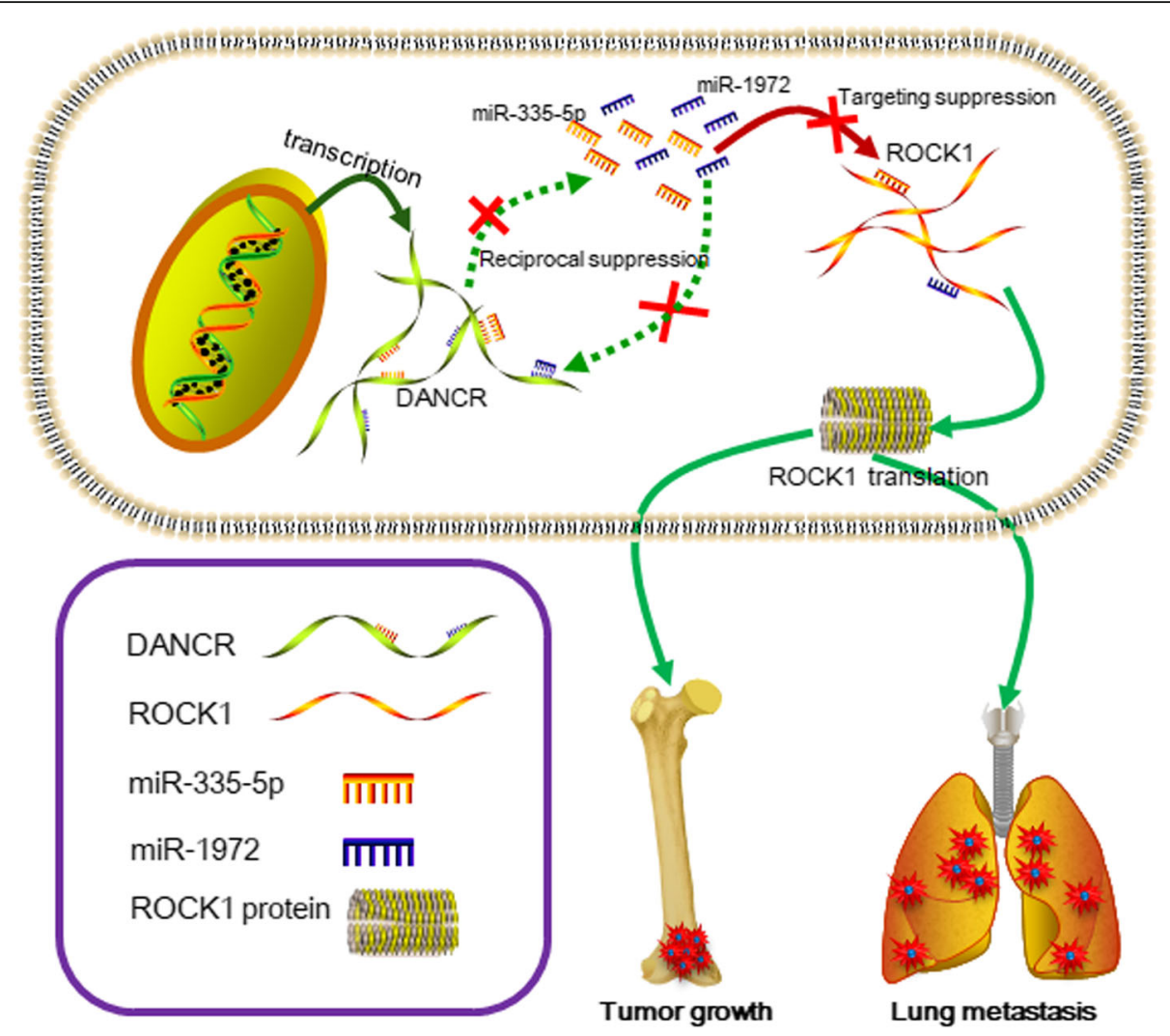

Fig. 7 Schematic diagram of mechanism on this research. DANCR promotes ROCK1-mediated proliferation and metastasis via decoying of miR-335-5p and miR-1972 in osteosarcoma

ROCK1 and miR-335-5p/miR-1972 in molecular level, respectively. Convincingly, the constructed luciferase assay and RIP binding assay certified that both DANCR and ROCK1 were the targets of miR-335-5p/miR-1972. In addition, an executed antisense experiment strongly stated that it was wild DANCR cDNA but not mutant DNACR cDNA could reverse the suppressive effect on proliferation and metastasis which was caused by elevation of miR-335-5p or miR-1972. Finally, in an in vivo animal study, we verified again that DNACR positively triggered tumor growth and lung metastasis in mice. Together, as the schematic diagram presented in Fig. 7, we drew the conclusion that DANCR promoted ROCK1mediated proliferation and metastasis by acting as a ceRNA via decoying of miR-335-5p and miR-1972 in osteosarcoma.

The development and progression of osteosarcoma is a very complicated biological process which including an ocean of molecules and multiple mechanisms. The ceRNA network which are comprised by DNACR, miR335-5p/miR-1972 and ROCK1 is a hidden corner of iceberg in osteosarcoma. We have reasons to believe that numerous genes and downstream targets of DANCR are still worthy of further exploring.

\section{Conclusions}

The present study firstly demonstrated that DANCR could decoy two miRNAs - miR-335-5p and miR-1972 to facilitate ROCK1-mediated proliferation and metastasis via a ceRNA network. This provide a novel target and a better understanding for possibly potential mechanism of pathogenesis and molecular therapeutic strategy for osteosarcoma.

\section{Additional files}

Additional file 1: Table S1. The sequences of primers used in this work. (DOCX $18 \mathrm{~kb}$ )

Additional file 2: Figure S2. The silenced efficiencies of DANCR and ROCK1 knock down plasmids were qualified by a qRT-PCR assays, and DANCR shRNA-01 as well as ROCK1 shRNA-01 were selected in the following RNAi experiments. (JPG 722 kb)

Additional file 3: Figure S1. DIANA TOOLS was applied to predict the potential miRNAs that might interact with DANCR, and the top 18 miRNAs were demonstrated for their higher theoretical binding scores (higher than 0.900). (JPG 3899 kb)

\section{Abbreviations}

3'-UTR: 3'-untranslated region; CeRNA: Competing endogenous RNA; DANCR: Differentiation antagonizing non-protein coding RNA;

LncRNAs: Long non-coding RNAs; MiRNAs: microRNAs; MREs: miRNA 
response elements; RIP assay: RNA immunoprecipitation assay; ROCK1: Rho associated coiled-coil containing protein kinase 1

\section{Funding}

This work was supported by grants from National Natural Science Foundation of China (No 81502333).

\section{Authors' contributions}

YW and XZ conceived the experiments; NW, YZ and ZL performed the experiments; YW, WZ and ST analyzed the data; YW and XZ wrote the manuscript. All authors read and approved the final manuscript.

\section{Ethics approval and consent to participate}

All osteosarcoma tissues and matched para-tumor tissues were in accordance with the ethical guidelines of Liaoning Cancer Hospital \& Institute and the Helsinki declaration. The ethics consents were signed by each patient before the study. All patients agreed that the data from their samples could be used for experimental studies and paper presentations.

\section{Competing interests}

The authors declare that they have no competing interests.

\section{Publisher's Note}

Springer Nature remains neutral with regard to jurisdictional claims in published maps and institutional affiliations.

\section{Author details}

${ }^{1} 4$ th Department of Orthopedic Surgery, Central Hospital affiliated to Shenyang Medical College, No.5, South Seven West Road, Tiexi District, Shenyang, Liaoning 110024, People's Republic of China. ${ }^{2}$ Department of Surgical Oncology, Central Hospital affiliated to Shenyang Medical College, Shenyang, People's Republic of China. ${ }^{3}$ 2nd Department of Cardiology, Central Hospital affiliated to Shenyang Medical College, Shenyang, People's Republic of China. ${ }^{4}$ Institute for Cardiovascular Prevention, Ludwig-Maximilians-University, Munich, Germany. ${ }^{5} 1$ st Department of Hand Surgery, Central Hospital affiliated to Shenyang Medical College, Shenyang, People's Republic of China. 'Department of Pathology, Liaoning Cancer Hospital \& Institute, Shenyang, People's Republic of China. ${ }^{7}$ Department of Nuclear Medicine, The First Affiliated Hospital of Dalian Medical University, Dalian 116011, People's Republic of China.

\section{Received: 17 February 2018 Accepted: 25 April 2018}

\section{Published online: 12 May 2018}

\section{References}

1. Berner K, Johannesen TB, Berner A, Haugland HK, Bjerkehagen B, Bohler PJ, Bruland OS. Time-trends on incidence and survival in a nationwide and unselected cohort of patients with skeletal osteosarcoma. Acta oncologica (Stockholm, Sweden). 2015;54(1):25-33.

2. Sampo M, Koivikko M, Taskinen M, Kallio P, Kivioja A, Tarkkanen M, Bohling T. Incidence, epidemiology and treatment results of osteosarcoma in Finland - a nationwide population-based study. Acta oncologica (Stockholm, Sweden). 2011;50(8):1206-14.

3. Ottaviani G, Jaffe N. The epidemiology of osteosarcoma. Cancer Treat Res. 2009:152:3-13.

4. Han Li C, Chen Y. Small and long non-coding RNAs: novel targets in perspective cancer therapy. Curr Genomics. 2015;16(5):319-26.

5. Wang $Y$, Zhang $Y$, Yang T, Zhao W, Wang N, Li P, Zeng X, Zhang W. Long non-coding RNA MALAT1 for promoting metastasis and proliferation by acting as a ceRNA of miR-144-3p in osteosarcoma cells. Oncotarget. 2017; 8(35):59417-34

6. Yang G, Lu X, Yuan L. LncRNA: a link between RNA and cancer. Biochim Biophys Acta. 2014;1839(11):1097-109.

7. Li Z, Tang Y, Xing W, Dong W, Wang Z. LncRNA, CRNDE promotes osteosarcoma cell proliferation, invasion and migration by regulating Notch1 signaling and epithelial-mesenchymal transition. Exp Mol Pathol. 2017;104(1):19-25

8. Liu H, Zhou G, Fu X, Cui H, Pu G, Xiao Y, Sun W, Dong X, Zhang L, Cao S, Li $G$, Wu $X$, Yang $X$. Long noncoding RNA TUG1 is a diagnostic factor in lung adenocarcinoma and suppresses apoptosis via epigenetic silencing of BAX Oncotarget. 2017;8(60):101899-910.
9. $\quad$ Tang WG, Hu B, Sun HX, Sun QM, Sun C, Fu PY, Yang ZF, Zhang X, Zhou $\mathrm{CH}$, Fan J, Ren N, Xu Y. Long non-coding RNA00364 represses hepatocellular carcinoma cell proliferation via modulating P-STAT3-IFIT2 signaling axis. Oncotarget. 2017:8(60):102006-19.

10. Wang K, Jin W, Song Y, Fei X. LncRNA RP11-436H11.5, functioning as a competitive endogenous RNA, upregulates BCL-W expression by sponging miR-335-5p and promotes proliferation and invasion in renal cell carcinoma. Mol Cancer. 2017; 16(1):166.

11. Yoon JH, You BH, Park CH, Kim YJ, Nam JW and Lee SK. The long noncoding RNA LUCAT1 promotes tumorigenesis by controlling ubiquitination and stability of DNA methyltransferase 1 in esophageal squamous cell carcinoma. Cancer letters. 2018;417:47-57.

12. Salmena $L$, Poliseno $L$, Tay $Y$, Kats L, Pandolfi PP. A ceRNA hypothesis: the Rosetta stone of a hidden RNA language? Cell. 2011;146(3):353-8.

13. Wang $Y$, Wang N, Zeng X, Sun J, Wang G, Xu H, Zhao W. MicroRNA-335 and its target Rock1 synergistically influence tumor progression and prognosis in osteosarcoma. Oncol Lett. 2017:13(5):3057-65.

14. Wang Y, Zhao W, Fu Q. miR-335 suppresses migration and invasion by targeting ROCK1 in osteosarcoma cells. Mol Cell Biochem. 2013;384(12):105-11.

15. Chen C, Ridzon DA, Broomer AJ, Zhou Z, Lee DH, Nguyen JT, Barbisin M, Xu NL, Mahuvakar VR, Andersen MR, Lao KQ, Livak KJ, Guegler KJ. Real-time quantification of microRNAs by stem-loop RT-PCR. Nucleic Acids Res. 2005; 33(20):e179

16. Wang Y, Yang T, Liu Y, Zhao W, Zhang Z, Lu M, Zhang W. Decrease of miR-195 promotes chondrocytes proliferation and maintenance of chondrogenic phenotype via targeting FGF-18 pathway. Int J Mol Sci. 2017;18(5). https:/doi. org/10.3390/ijms18050975.

17. Cai H, Liu X, Zheng J, Xue Y, Ma J, Li Z, Xi Z, Li Z, Bao M, Liu Y. Long noncoding RNA taurine upregulated 1 enhances tumor-induced angiogenesis through inhibiting microRNA-299 in human glioblastoma. Oncogene. 2017; 36(3):318-31.

18. Huang J, Shi Y, Li H, Yang M, Liu G. MicroRNA-144 acts as a tumor suppressor by targeting Rho-associated coiled-coil containing protein kinase 1 in osteosarcoma cells. Mol Med Rep. 2015;12(3):4554-9.

19. Ell B, Kang Y. MicroRNAs as regulators of bone homeostasis and bone metastasis. Bonekey Rep. 2014;3:549.

20. Kafchinski LA, Jones KB. MicroRNAs in osteosarcomagenesis. Adv Exp Med Biol. 2014;804:119-27.

21. Sampson VB, Yoo S, Kumar A, Vetter NS, Kolb EA. MicroRNAs and potential targets in osteosarcoma: review. Front Pediatr. 2015;3:69.

22. Cai L, LV J, Zhang Y, Li J, Wang Y, Yang $H$. The IncRNA HNF1A-AS1 is a negative prognostic factor and promotes tumorigenesis in osteosarcoma. J Cell Mol Med. 2017;21(11):2654-62.

23. Jia D, Niu Y, Li D, Liu Z. LnCRNA C2dat1 promotes cell proliferation, migration, and invasion by targeting MiR-34a-5p in osteosarcoma cells. Oncol Res. 2017; https://doi.org/10.3727/096504017X15024946480113.

24. Li N, Sun ZH, Fang M, Xin JY, Wan CY. Long non-coding RNA ZFAS1 sponges miR-486 to promote osteosarcoma cells progression and metastasis in vitro and vivo. Oncotarget. 2017;8(61):104160-70.

25. Yang C, Wang G, Yang J, Wang L. Long noncoding RNA NBAT1 negatively modulates growth and metastasis of osteosarcoma cells through suppression of miR-21. Am J Cancer Res. 2017:7(10):2009-19.

26. Yuan SX, Wang J, Yang F, Tao QF, Zhang J, Wang LL, Yang Y, Liu H, Wang ZG, Xu QG, Fan J, Liu L, Sun SH, Zhou WP. Long noncoding RNA DANCR increases stemness features of hepatocellular carcinoma by derepression of CTNNB1. Hepatology (Baltimore, Md). 2016;63(2):499-511.

27. Jia J, Li F, Tang XS, Xu S, Gao Y, Shi Q, Guo W, Wang X, He D, Guo P. Long noncoding RNA DANCR promotes invasion of prostate cancer through epigenetically silencing expression of TIMP2/3. Oncotarget. 2016;7(25): 37868-81.

28. Jiang N, Wang X, Xie X, Liao Y, Liu N, Liu J, Miao N, Shen J, Peng T. IncRNA DANCR promotes tumor progression and cancer stemness features in osteosarcoma by upregulating AXL via miR-33a-5p inhibition. Cancer Lett. 2017;405:46-55.

29. Derrien T, Johnson R, Bussotti G, Tanzer A, Djebali S, Tilgner H, Guernec G, Martin D, Merkel A, Knowles DG, Lagarde J, Veeravalli L, Ruan X, Ruan Y, Lassmann T, Carninci P, et al. The GENCODE v7 catalog of human long noncoding RNAs: analysis of their gene structure, evolution, and expression. Genome Res. 2012;22(9):1775-89.

30. Chen DL, Lu YX, Zhang JX, Wei XL, Wang F, Zeng ZL, Pan ZZ, Yuan YF, Wang FH, Pelicano H, Chiao PJ, Huang P, Xie D, Li YH, Ju HQ, Xu RH. Long 
non-coding RNA UICLM promotes colorectal cancer liver metastasis by acting as a ceRNA for microRNA-215 to regulate ZEB2 expression. Theranostics. 2017;7(19):4836-49.

31. Li H, Chen S, Liu J, Guo X, Xiang X, Dong T, Ran P, Li Q, Zhu B, Zhang X, Wang D, Xiao C, Zheng S. Long non-coding RNA PVT1-5 promotes cell proliferation by regulating miR-126/SLC7A5 axis in lung cancer. Biochem. Biophys Res Commun. 2017; https://doi.org/10.1016/j.bbrc.2017.12.114.

32. Lin J, Shi Z, Yu Z, He Z. LncRNA HIF1A-AS2 positively affects the progression and EMT formation of colorectal cancer through regulating miR-129-5p and DNMT3A. Biomed Pharmacother. 2017;98:433-9.

33. Ma J, Fan Y, Feng T, Chen F, Xu Z, Li S, Lin Q, He X, Shi W, Liu Y, Cao X, Zhu $B$, Liu Z. HOTAIR regulates HK2 expression by binding endogenous miR-125 and miR-143 in oesophageal squamous cell carcinoma progression. Oncotarget. 2017;8(49):86410-22.

34. Xie CR, Wang F, Zhang S, Wang FQ, Zheng S, Li Z, Lv J, Qi HQ, Fang QL, Wang XM, Yin ZY. Long noncoding RNA HCAL facilitates the growth and metastasis of hepatocellular carcinoma by acting as a ceRNA of LAPTM4B. Mol Ther Nucleic Acids. 2017;9:440-51.

35. Yang T, Zhou H, Liu P, Yan L, Yao W, Chen K, Zeng J, Li H, Hu J, Xu H, Ye Z. IncRNA PVT1 and its splicing variant function as competing endogenous RNA to regulate clear cell renal cell carcinoma progression. Oncotarget. 2017:8(49):85353-67.

36. Zhao L, Liu B. Identification of potential prognostic ceRNA module biomarkers in patients with pancreatic adenocarcinoma. Oncotarget. 2017; 8(55):94493-504.

37. Wang Y, Yang T, Zhang Z, Lu M, Zhao W, Zeng X, Zhang W. Long noncoding RNA TUG1 promotes migration and invasion by acting as a ceRNA of miR-335-5p in osteosarcoma cells. Cancer Sci. 2017;108(5):859-67.

38. Li CH, Yu TB, Qiu HW, Zhao X, Zhou CL, Qi C. miR-150 is downregulated in osteosarcoma and suppresses cell proliferation, migration and invasion by targeting ROCK1. Oncol Lett. 2017;13(4):2191-7.

39. Liu Y, Liu J, Wang L, Yang X, Liu X. MicroRNA195 inhibits cell proliferation, migration and invasion in laryngeal squamous cell carcinoma by targeting ROCK1. Mol Med Rep. 2017;16(5):7154-62.

40. Qin Z, Wei X, Jin N, Wang Y, Zhao R, Hu Y, Yan W, Li J, Zhou Q. MiR-199a targeting ROCK1 to affect kidney cell proliferation, invasion and apoptosis. Artif Cells Nanomed Biotechnol. 2017:1-6. https://doi.org/10.1080/21691401. 2017.1396224

41. Zhan Y, Zheng N, Teng F, Bao L, Liu F, Zhang M, Guo M, Guo W, Ding G, Wang Q. MiR-199a/b-5p inhibits hepatocellular carcinoma progression by post-transcriptionally suppressing ROCK1. Oncotarget. 2017;8(40):67169-80.

42. Han C, Wang W. MicroRNA-129-5p suppresses cell proliferation, migration and invasion via targeting ROCK1 in osteosarcoma. Mol Med Rep. 2018; 17(3):4777-84

43. Maskey N, Li D, Xu H, Song H, Wu C, Hua K, Song J, Fang L. MicroRNA-340 inhibits invasion and metastasis by downregulating ROCK1 in breast cancer cells. Oncol Lett. 2017;14(2):2261-7.

44. Wu S, Ai N, Liu Q, Zhang J. MicroRNA448 inhibits the progression of retinoblastoma by directly targeting ROCK1 and regulating PI3K/AKT signalling pathway. Oncol Rep. 2018;39(5):2402-12.

\section{Ready to submit your research? Choose BMC and benefit from:}

- fast, convenient online submission

- thorough peer review by experienced researchers in your field

- rapid publication on acceptance

- support for research data, including large and complex data types

- gold Open Access which fosters wider collaboration and increased citations

- maximum visibility for your research: over $100 \mathrm{M}$ website views per year

At BMC, research is always in progress.

Learn more biomedcentral.com/submissions 